\title{
Real-time estimation of daily physical activity intensity by a triaxial accelerometer and a gravity-removal classification algorithm
}

\author{
Kazunori Ohkawara $^{1,2 *}$, Yoshitake Oshima ${ }^{3}$, Yuki Hikihara ${ }^{4}$, Kazuko Ishikawa-Takata ${ }^{1}$, Izumi Tabata ${ }^{1,5}$ \\ and Shigeho Tanaka ${ }^{1}$ \\ ${ }^{1}$ Health Promotion and Exercise Program, National Institute of Health and Nutrition, 1-23-1 Toyama, Shinjuku-ku, Tokyo \\ 162-8636, Japan \\ ${ }^{2}$ Center for Human Nutrition, University of Colorado Denver, Denver, CO, USA \\ ${ }^{3}$ Research and Development Department, Omron Healthcare Company Limited, Kyoto, Japan \\ ${ }^{4}$ Faculty of Engineering, Chiba Institute of Technology, Narashino, Japan \\ ${ }^{5}$ Faculty of Sport and Health Sciences, Ritsumeikan University, Shiga, Japan
}

(Received 14 June 2010 - Revised 18 November 2010 - Accepted 19 November 2010 - First published online 25 January 2011)

\section{Abstract}

We have recently developed a simple algorithm for the classification of household and locomotive activities using the ratio of unfiltered to filtered synthetic acceleration (gravity-removal physical activity classification algorithm, GRPACA) measured by a triaxial accelerometer. The purpose of the present study was to develop a new model for the immediate estimation of daily physical activity intensities using a triaxial accelerometer. A total of sixty-six subjects were randomly assigned into validation $(n$ 44) and cross-validation ( $n$ 22) groups. All subjects performed fourteen activities while wearing a triaxial accelerometer in a controlled laboratory setting. During each activity, energy expenditure was measured by indirect calorimetry, and physical activity intensities were expressed as metabolic equivalents (MET). The validation group displayed strong relationships between measured MET and filtered synthetic accelerations for household ( $r$ 0.907, $P<0.001)$ and locomotive $(r$ 0.961, $P<0.001)$ activities. In the cross-validation group, two GRPACA-based linear regression models provided highly accurate MET estimation for household and locomotive activities. Results were similar when equations were developed by non-linear regression or sex-specific linear or non-linear regressions. Sedentary activities were also accurately estimated by the specific linear regression classified from other activity counts. Therefore, the use of a triaxial accelerometer in combination with a GRPACA permits more accurate and immediate estimation of daily physical activity intensities, compared with previously reported cutoff classification models. This method may be useful for field investigations as well as for self-monitoring by general users.

Key words: Non-exercise activity thermogenesis: Accelerometry: Household activity: Locomotive activity: Metabolic equivalents

Low physical activity (PA) levels in daily life are probably correlated with obesity and other diseases ${ }^{(1)}$. According to the International Association for the Study of Obesity, prevention of weight regain in formerly obese individuals requires 60-90 min of daily moderate activity or lesser amounts of vigorous activity, with $45-60 \mathrm{~min}$ of daily moderate activity required to prevent the transition to overweight or obese $^{(2)}$. In addition to exercise, non-exercise activity thermogenesis, a much larger part of daily PA, may also contribute to obesity prevention ${ }^{(3,4)}$. Therefore, assessment of the type, quantity and intensity of PA is important for the development of strategies to prevent obesity and chronic diseases. However, accurate methods for the measurement of energy expenditures (EE) induced by various PA under free-living conditions are still under consideration.

At present, several methods are used for the measurement of $\mathrm{EE}$ in a field setting ${ }^{(5,6)}$. The doubly labelled water method displays high accuracy for the measurement of $24 \mathrm{~h}$ EE under free-living conditions. However, this method can only evaluate total $\mathrm{EE}$ and cannot provide day-to-day or minute-by-minute variations. Although questionnaires could individually measure PA intensity and $\mathrm{EE}$ (as value by intensity $\times$ time) in addition to the PA type, the accuracy of these methods is not sufficient ${ }^{(7)}$. On the other hand, accelerometers are objective, small, non-invasive tools for measuring PA intensity and EE, with the potential to measure locomotive

Abbreviations: ACC $_{\text {fil }}$, filtered synthetic acceleration; ACC $_{\text {unfil }}$, unfiltered synthetic acceleration; EE, energy expenditure; GRPACA, gravity-removal physical activity classification algorithm; MET, metabolic equivalent; PA, physical activity.

*Corresponding author: K. Ohkawara, fax +8133204 1761, email ohkawara@nih.go.jp 
as well as household activities ${ }^{(8-10)}$. Furthermore, activity monitors such as accelerometers or pedometers may serve as useful tools for promoting active life behaviour ${ }^{(11,12)}$.

At the least, uniaxial and triaxial accelerometers can accurately estimate the intensity of ambulatory activities ${ }^{(13-15)}$. However, the intensities of household activities such as vacuuming and sweeping cannot be accurately estimated by accelerometers, possibly leading to underestimation of total $\mathrm{EE}$ by algorithms based on locomotive activities ${ }^{(14)}$. Indeed, different relationships between counts per minute and metabolic equivalents (MET) observed for locomotive $v$. household activities led to MET underestimation for household activities ${ }^{(13-15)}$. Time spent in sedentary and light activities is also underestimated by locomotion-based equations ${ }^{(16)}$. Therefore, accurate MET estimation for household and sedentary activities is required in addition to locomotive activity.

Recently, several studies have attempted to discriminate between PA types using accelerometer counts ${ }^{(17-26)}$. Although these algorithms have improved accuracy for estimating the MET of various activities compared with single regression models, some limitations remain: percentage of correct classification was slightly lower in some types of $\mathrm{PA}^{(21,22)}$; multiple sensors make it difficult to continuously wear the device on the body ${ }^{(26)}$; estimation is a complex procedure requiring large amounts of data, a barrier for applied researchers as well as for the general public. An accelerometer-based algorithm that accurately and immediately estimates PA intensity would be a useful tool for assessing PA in free-living conditions, as well as for promoting active life behaviour in general users. We have recently developed a simple but accurate algorithm for the classification of locomotive and household activities, using the ratio of unfiltered to filtered synthetic acceleration ( $\left.\mathrm{ACC}_{\text {unfil }} / \mathrm{ACC}_{\mathrm{fil}}\right)$ combined with a gravityremoval PA classification algorithm (GRPACA $)^{(27)}$. A correct classification percentage of almost $100 \%$ was achieved during our selected activities. Furthermore, we have confirmed the separation of sedentary activities from both locomotive and household activities by accelerometer counts. Therefore, the purpose of the present study was to develop a new model for instantly estimating the intensity of daily PA using a triaxial accelerometer.

\section{Subjects and methods}

\section{Subjects}

A total of sixty-six subjects (thirty-one males and thirty-five females) volunteered to participate in the present study. The present study was conducted according to the guidelines laid down in the Declaration of Helsinki, and all procedures involving human subjects were approved by the Ethical Committee of the National Institute of Health and Nutrition in Tokyo, Japan. Subjects were excluded from the study if they had any contraindications to exercise, or if they were physically unable to complete the activities. Descriptive characteristics of the study subjects are presented in Table 1. Subjects were randomly assigned into validation ( $n$ 44) and crossvalidation ( $n$ 22) groups. Before measurement, the purpose

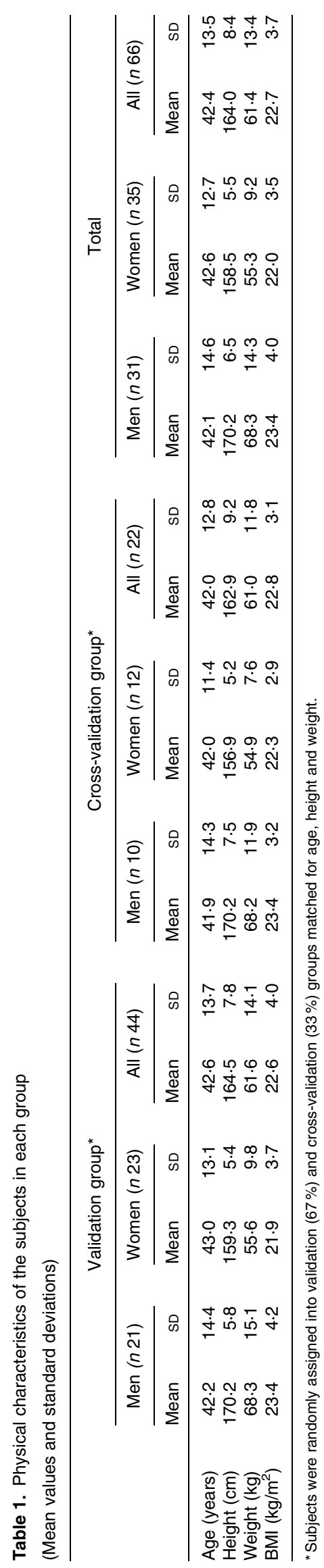


and procedure of the study were explained in detail. Informed consent was signed by all subjects.

\section{Anthropometric measurements}

Before performing PA, body weight was measured by a digital scale to the nearest $0 \cdot 1 \mathrm{~kg}$, with the subjects dressed in light clothing. Barefoot standing height was measured to the nearest $0 \cdot 1 \mathrm{~cm}$ using a wall-mounted stadiometer (YL-65S; Yagami, Nagoya, Japan). BMI was calculated as body weight (kg) divided by height squared $\left(\mathrm{m}^{2}\right)$.

\section{Experimental protocol}

Fasting subjects visited the laboratory in the morning of the experimental day. After anthropometric measurements, they performed fourteen activities with a facemask and Douglas bag while wearing a triaxial accelerometer on the left side of the waist. The selected activities were as follows: (1) sedentary activity - resting in the supine position as BMR, resting in the sitting position as RMR and personal computer work; (2) household activity - laundry, dishwashing, moving a small load ( $5 \mathrm{~kg}$ ) and vacuuming; (3) locomotive activity - slow walking $(3.3 \mathrm{~km} / \mathrm{h})$, normal walking $(4.2 \mathrm{~km} / \mathrm{h})$, brisk walking $(6.0 \mathrm{~km} / \mathrm{h})$, normal walking while carrying a bag $(3 \mathrm{~kg})$ in the hand, jogging $(8.4 \mathrm{~km} / \mathrm{h})$ on a track, and ascending and descending stairs at personal normal speeds without using handrails. These activities were chosen as representative activities of daily life, based on our observations in a preliminary study using the activity records of other subjects. The subjects were permitted to consume only drinking-water during the experiment. They were instructed to lie down quietly for $30 \mathrm{~min}$, and then BMR was measured for two periods of $10 \mathrm{~min}$, followed by RMR measurement for $10 \mathrm{~min}$. Subsequently, the other activities were performed for 3-7 min. The entire experimental protocol took each subject about $4.5 \mathrm{~h}$ to complete, and there was enough rest between activities to eliminate any carry-over effect from one activity to another. Each subject performed the experiment following the same schedule. The expired air for the subject in each activity was collected under a steady state. We defined the beginning of the steady state as $2-3$ min after starting an activity, depending on the activity intensity ${ }^{(28)}$. This experimental protocol has previously been described in detail ${ }^{(27)}$.

\section{Indirect calorimetry}

During each activity, the subject's expired air was collected in a Douglas bag. Expired $\mathrm{O}_{2}$ and $\mathrm{CO}_{2}$ gas concentrations were measured by MS (ARCO-1000; Arco System, Kashiwa, Japan), and gas volume was determined using a certified dry gas meter (DC-5; Shinagawa, Tokyo, Japan). For each measurement, the gas analyser was initially calibrated using a certified gas mixture and atmospheric air. EE was estimated from $\mathrm{VO}_{2}$ and $\mathrm{VCO}_{2}$ using Weir's equation ${ }^{(29)}$. MET values as reference were calculated as EE during the activities divided by the measured RMR.

\section{Triaxial accelerometer}

We used a triaxial accelerometer with 4 GB of memory consisting of Micro Electro Mechanical Systems-based accelerometers (LIS3LV02DQ; ST-Microelectronics, Geneva, Switzerland), which respond to both acceleration due to movement and gravitational acceleration. The sensor was built into a plastic case without a liquid crystal display and was designed to be clipped to a waist belt (size: $80 \times 50 \times 20 \mathrm{~mm}$; weight: approximately $60 \mathrm{~g}$ including batteries). Anteroposterior ( $x$ axis), mediolateral ( $y$-axis) and vertical ( $z$-axis) acceleration measurements were obtained during each activity at a rate of $32 \mathrm{~Hz}$ to $12 \mathrm{bit}$ accuracy. The range of the acceleration data of each axis is $\pm 6 \mathrm{G}$, resulting in a resolution of $3 \mathrm{mG}$. The acceleration data were uploaded to a personal computer.

The signals obtained from the triaxial accelerometer were processed in the following way. Each of the three signals from the triaxial accelerometer was passed through a highpass filter with a cut-off frequency of $0.7 \mathrm{~Hz}$, in order to remove the gravitational acceleration component from the signal. We calculated the synthetic acceleration of all three axes (vector magnitude $\sqrt{x^{2}+y^{2}+z^{2}}$ ) using signals before and after high-pass filtering. Then, the ratio of $\mathrm{ACC}_{\text {unfil }}$ to $\mathrm{ACC}_{\text {fil }}$ was calculated. The acceleration signals, calculated as the average of the absolute value of the accelerometer output of each axis from $10 \mathrm{~s}$ epochs at the middle of each activity, were processed to various acceleration output variables. In our previous study, we reported the algorithm for the classification of household and locomotive activities by the $\mathrm{ACC}_{\text {unfil }}: \mathrm{ACC}_{\text {fil }}$ ratio which resulted in almost $100 \%$ correct demarcation for our eleven selected activities ${ }^{(27)}$

A commercial product (Activity Style Pro HJA-350IT; Omron Healthcare, Kyoto, Japan) has been developed from the prototype accelerometer that we made in the present study. This commercial device measures $74 \times 46 \times 34 \mathrm{~mm}$ and weighs $60 \mathrm{~g}$, including batteries. The liquid crystal display in this device has several modes that provide different types of information: (1) a research mode that provides no information; (2) a mode that displays step counts; (3) a mode that displays realtime MET intensity. Both devices are shown in Fig. 1.

\section{Statistical analysis}

All values are presented as means and standard deviations. Differences are considered to be statistically significant if the $P$ value is less than $0 \cdot 05$. The relationship between measured MET and the $\mathrm{ACC}_{\text {fil }}$ count in the validation group was evaluated by Pearson's correlation coefficient $(r)$ and the standard error of the estimate. Linear and non-linear regression models were used in the validation group to develop equations to predict MET based on the intensity of PA, as measured by the $\mathrm{ACC}_{\mathrm{fil}}$ count. Differences between measured and estimated MET in the cross-validation group were assessed by one-way ANOVA followed by Dunnett's post hoc test or a paired $t$ test. Bland-Altman plots were used to graphically show the variability in individual error scores in the cross-validation group ${ }^{(30)}$. All statistical analyses were 
(a)

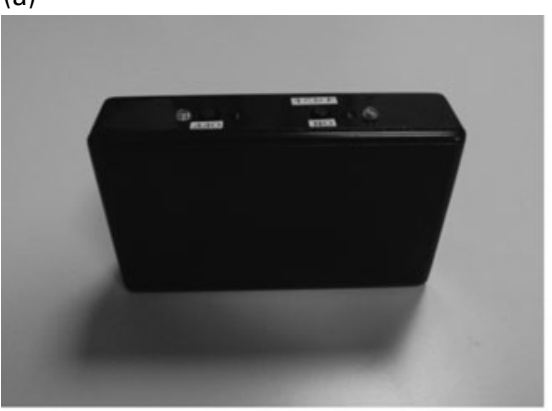

(c)

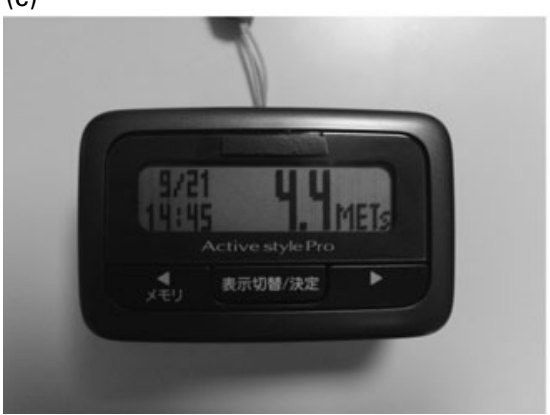

(b)

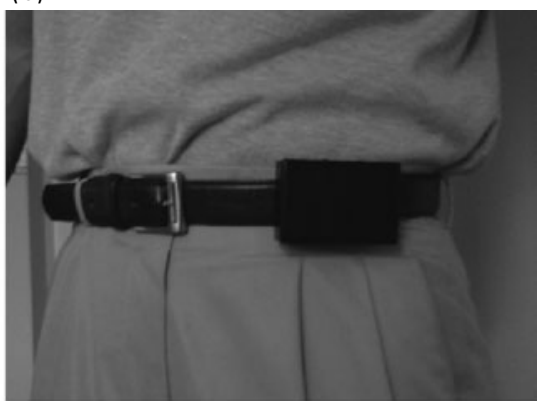

(d)

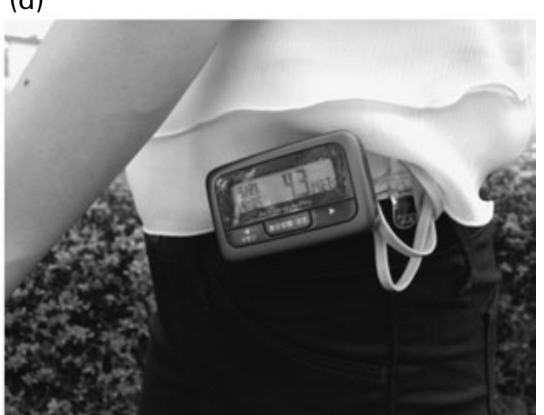

Fig. 1. Prototype accelerometer used in the present study and a commercial accelerometer based on the algorithm developed in the present study. (a) Prototype accelerometer that was used to perform all measurements; (b) subjects wore the prototype accelerometer on the waist with a clip during the entire protocol; (c) commercial accelerometer based on the algorithm that was developed in the present study; (d) real-time metabolic equivalents (MET) are shown on the liquid crystal display (LCD) of the commercial accelerometer (the LCD can also show step counts).

Table 2. Energy expenditure, metabolic equivalents (MET), accelerations and acceleration ratios for each activity in the validation group (Mean values and standard deviations, $n$ 44)

\begin{tabular}{|c|c|c|c|c|c|c|c|c|c|c|c|c|}
\hline & \multicolumn{2}{|c|}{$\begin{array}{c}\text { Energy } \\
\text { expenditure } \\
(\mathrm{kJ} / \mathrm{min})\end{array}$} & \multicolumn{2}{|c|}{$\mathrm{MET}^{*}$} & \multicolumn{2}{|c|}{ MET† } & \multicolumn{2}{|c|}{$\begin{array}{l}\text { Unfiltered } \\
\text { synthetic } \\
\text { acceleration } \\
(\mathrm{mG})\end{array}$} & \multicolumn{2}{|c|}{$\begin{array}{l}\text { Filtered } \\
\text { synthetic } \\
\text { acceleration } \\
(\mathrm{mG})\end{array}$} & \multicolumn{2}{|c|}{$\begin{array}{l}\text { Ratio of } \\
\text { unfiltered } \\
\text { synthetic } \\
\text { acceleration } \\
\text { to filtered } \\
\text { synthetic } \\
\text { acceleration }\end{array}$} \\
\hline & Mean & SD & Mean & SD & Mean & SD & Mean & SD & Mean & SD & Mean & SD \\
\hline \multicolumn{13}{|l|}{ Light activity } \\
\hline $\begin{array}{l}\text { Resting in the sitting } \\
\text { position }(n 44)\end{array}$ & $4 \cdot 142$ & 0.79 & \multicolumn{2}{|c|}{-} & \multicolumn{2}{|c|}{-} & $5 \cdot 6$ & $1 \cdot 8$ & $2 \cdot 6$ & 0.6 & $2 \cdot 15$ & 0.63 \\
\hline $\begin{array}{l}\text { Resting in the supine } \\
\text { position ( } n 44)\end{array}$ & 3.765 & 0.79 & 0.91 & 0.05 & $0 \cdot 89$ & $0 \cdot 10$ & $4 \cdot 6$ & $2 \cdot 4$ & $2 \cdot 1$ & 0.7 & $2 \cdot 14$ & 0.88 \\
\hline $\begin{array}{l}\text { Personal computer } \\
\text { work ( } n \text { 42) }\end{array}$ & $4 \cdot 602$ & 1.00 & $1 \cdot 12$ & 0.08 & 1.08 & $0 \cdot 12$ & $10 \cdot 2$ & $3 \cdot 7$ & $5 \cdot 7$ & $1 \cdot 7$ & $1 \cdot 80$ & 0.37 \\
\hline \multicolumn{13}{|l|}{ Household activity } \\
\hline Laundry ( $n$ 44) & $9 \cdot 706$ & 2.59 & $2 \cdot 34$ & 0.37 & $2 \cdot 26$ & 0.31 & $154 \cdot 1$ & 38.4 & $50 \cdot 2$ & 11.5 & $3 \cdot 11$ & 0.57 \\
\hline Dishwashing ( $n$ 43) & $7 \cdot 614$ & $2 \cdot 01$ & 1.84 & 0.34 & 1.77 & 0.30 & $56 \cdot 8$ & $17 \cdot 9$ & $26 \cdot 3$ & $6 \cdot 7$ & $2 \cdot 20$ & 0.64 \\
\hline Moving a small load $(n 44)$ & $18 \cdot 32$ & 4.98 & 4.40 & 0.68 & $4 \cdot 27$ & 0.63 & $360 \cdot 5$ & 51.9 & $157 \cdot 1$ & 21.5 & $2 \cdot 32$ & 0.35 \\
\hline Vacuuming ( $n$ 42) & $12 \cdot 34$ & 3.01 & $2 \cdot 97$ & 0.52 & $2 \cdot 88$ & 0.53 & $153 \cdot 2$ & $34 \cdot 3$ & $82 \cdot 8$ & $24 \cdot 9$ & 1.92 & 0.39 \\
\hline \multicolumn{13}{|l|}{ Locomotive activity } \\
\hline Slow walking $(n 44)$ & 13.01 & 3.39 & $3 \cdot 12$ & 0.45 & 3.03 & 0.42 & 245.5 & 47.4 & $240 \cdot 1$ & $48 \cdot 1$ & 1.02 & 0.02 \\
\hline Normal walking $(n 44)$ & $15 \cdot 22$ & 3.81 & 3.67 & 0.55 & 3.56 & 0.49 & $320 \cdot 8$ & $48 \cdot 7$ & $313 \cdot 8$ & $48 \cdot 7$ & 1.02 & 0.02 \\
\hline Brisk walking $(n 44)$ & $19 \cdot 53$ & $5 \cdot 10$ & 4.70 & 0.76 & 4.56 & 0.75 & 428.4 & $69 \cdot 6$ & $426 \cdot 8$ & $72 \cdot 2$ & 1.01 & 0.02 \\
\hline $\begin{array}{l}\text { Walking while carrying a } \\
\text { bag }(n 44)\end{array}$ & $17 \cdot 90$ & $4 \cdot 14$ & $4 \cdot 33$ & 0.60 & $4 \cdot 20$ & 0.59 & 361.5 & $51 \cdot 8$ & $355 \cdot 7$ & 51.9 & 1.02 & 0.02 \\
\hline Jogging ( $n 44)$ & $39 \cdot 24$ & 9.37 & 9.42 & 0.98 & $9 \cdot 16$ & $1 \cdot 18$ & 974.2 & $118 \cdot 6$ & 954.0 & $116 \cdot 7$ & 1.02 & 0.02 \\
\hline Ascending stairs ( $n$ 39) & 31.54 & 6.86 & 7.64 & 0.75 & $7 \cdot 32$ & 0.61 & 232.4 & 29.5 & $220 \cdot 1$ & $29 \cdot 1$ & 1.06 & 0.04 \\
\hline Descending stairs ( $n$ 41) & $13 \cdot 38$ & 3.31 & 3.20 & 0.44 & 3.09 & 0.42 & 287.9 & $50 \cdot 6$ & 277.2 & $49 \cdot 4$ & 1.04 & 0.02 \\
\hline
\end{tabular}

${ }^{*}$ MET were calculated as energy expenditure for each activity divided by energy expenditure for resting in the sitting position.

† MET were calculated as energy expenditure for each activity divided by $4.184 \mathrm{~kJ} / \mathrm{kg}$ per $\mathrm{h}$. 


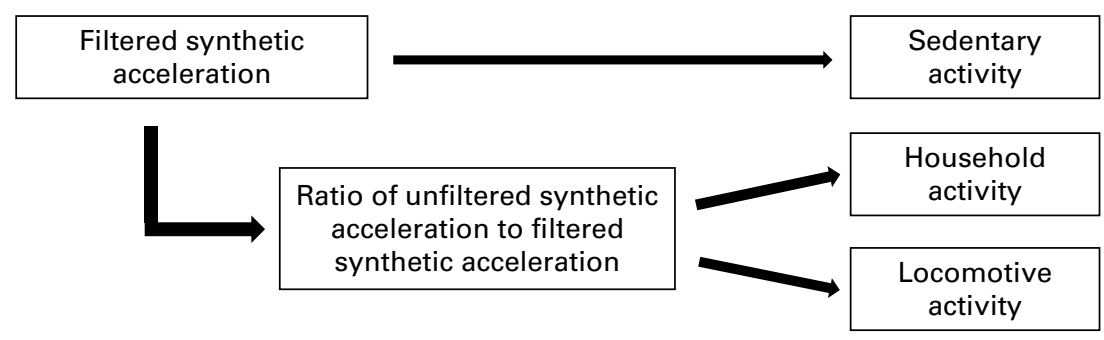

Fig. 2. Algorithm for the classification of three different activity types, using a triaxial accelerometer.

performed using SPSS version 15.0J for Windows (SPSS, Inc., Chicago, IL, USA).

\section{Results}

Data collected during the present study were analysed if both MET and ACC could be correctly measured during each activity. Mean EE, MET, $\mathrm{ACC}_{\mathrm{unfil}}, \mathrm{ACC}_{\mathrm{fil}}$ and the $\mathrm{ACC}_{\mathrm{unfil}}$ : $\mathrm{ACC}_{\mathrm{fi}}$ ratio for each activity are shown in Table 2 . As suggested previously $^{(16)}$, the one-regression models overestimate MET for light activity; we observed a similar result (data not shown). Therefore, we modelled the classification of our selected activities into three types of activities: sedentary, household and locomotive (Fig. 2). Sedentary activities are discriminated from household and locomotive activities, because ACC $_{\text {fil }}$ for sedentary activities was lower than for other activities. Household and locomotive activities are classified by the $\mathrm{ACC}_{\mathrm{unfil}}$ : ACC $_{\text {fil }}$ ratio according to our previous study $(1 \cdot 16)^{(27)}$.

Fig. 3 depicts the relationship between measured MET and $\mathrm{ACC}_{\text {fil }}$ during household and locomotive activities performed by the validation group. The correlation coefficients for locomotive ( $r 0.961, P<0.001)$, household $(r 0.907, P<0.001)$ and combined household and locomotive activities ( $r \quad 0.930$, $P<0.001)$ were high. We developed linear and non-linear regressions for estimating the intensities of household and locomotive activities; ascending and descending stairs were excluded from developing regressions, because the relationships between MET and $\mathrm{ACC}_{\text {fil }}$ for ascending and descending stairs differed from the relationship for the other locomotive activities (Table 3). As a result, the linear regression calculated

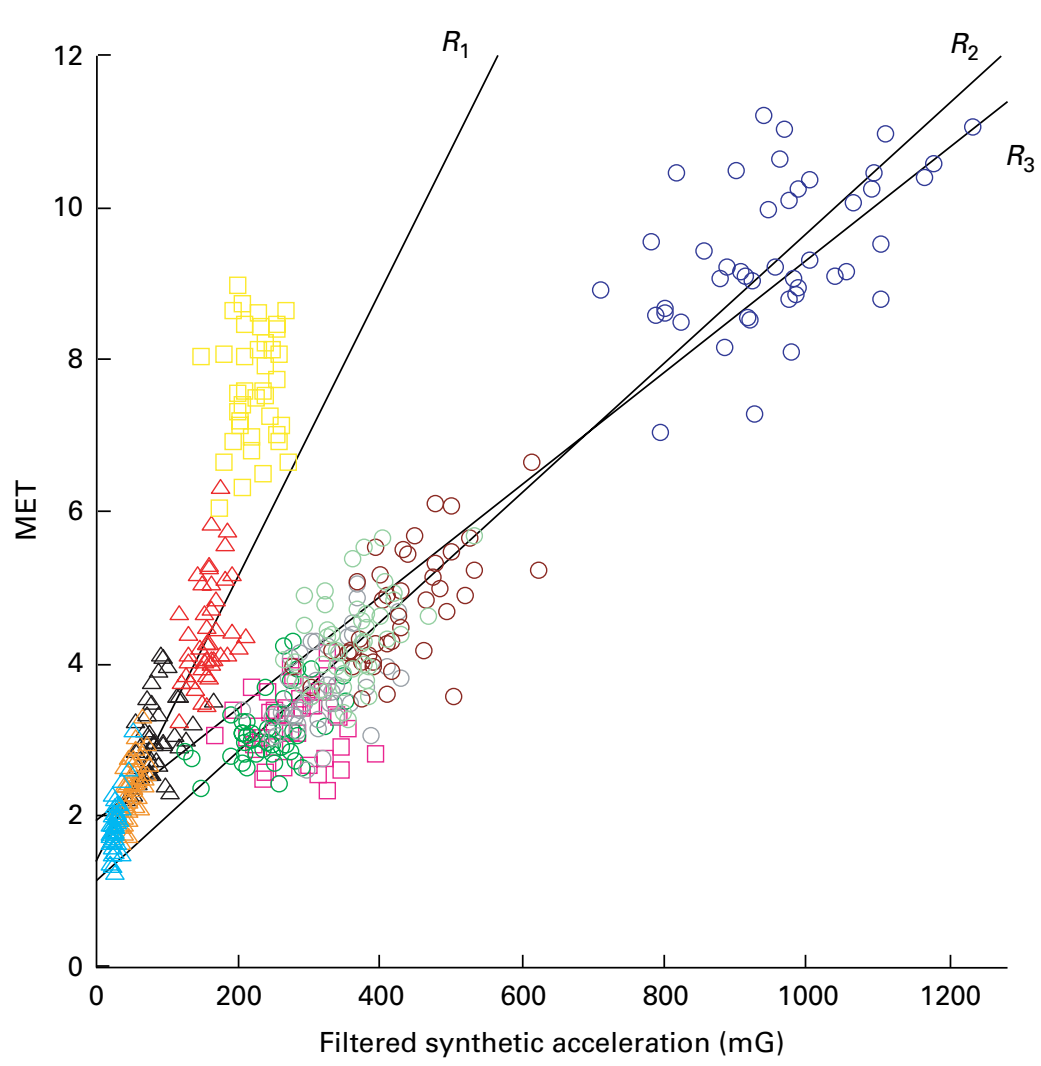

Fig. 3. Relationships between measured metabolic equivalents (MET) and filtered synthetic accelerations during locomotive and household activities in the validation group ( $n$ 44). $R_{1}(r 0.907, P<0.001)$, regression line for household activities only; $R_{2}(r 0.930, P<0.001)$, regression line for combined household and locomotive activities; $R_{3}(r 0.961, P<0.001)$, regression line for locomotive activity only. Ascending and descending stairs were removed from the regression analyses for $R_{1}, R_{2}$ and $R_{3}$. $\Delta$, Laundry; $\Delta$, dishwashing; $\Delta$, moving a small load; $\Delta$, vacuuming; $\bigcirc$, slow walking; $\bigcirc$, normal walking; $O$, brisk walking; $\bigcirc$, walking while carrying a bag; $\mathrm{O}$, jogging; $\square$, ascending stairs; $\square$, descending stairs. 
Table 3. Equations for estimating metabolic equivalents (MET) in locomotive and household activities by using filtered synthetic acceleration $\left(\mathrm{ACC}_{\mathrm{fil}}, \mathrm{mG}\right)$ in the validation group $(n 44)$

( $r$ Values and standard errors of the estimate (SEE))

\begin{tabular}{|c|c|c|c|}
\hline & Equation & $r$ & SEE (MET) \\
\hline \multicolumn{4}{|l|}{ Linear regression model } \\
\hline \multicolumn{4}{|l|}{ Model 1} \\
\hline Locomotive plus household activities & $M E T=1.9494+0.0074 \times A^{A C C_{f i l}}$ & $0.930^{*}$ & 0.804 \\
\hline \multicolumn{4}{|l|}{ Model 2} \\
\hline Locomotive activity only & $M E T=1.1372+0.0085 \times A^{A C C_{f i l}}$ & $0.961^{*}$ & 0.658 \\
\hline Household activity only & $\mathrm{MET}=1.4023+0.0188 \times \mathrm{ACC}_{\mathrm{fil}}$ & $0.907^{*}$ & 0.460 \\
\hline \multicolumn{4}{|l|}{ Non-linear regression model } \\
\hline \multicolumn{4}{|l|}{ Model 3} \\
\hline Locomotive activity only & $\mathrm{MET}=0.8944+0.0126 \times \mathrm{ACC}_{\mathrm{fil}}^{0.947}$ & $0.961^{*}$ & 0.657 \\
\hline Household activity only & $\mathrm{MET}=0.8149+0.1014 \times \mathrm{ACC}_{\mathrm{fil}}^{0.701}$ & $0.910^{*}$ & 0.453 \\
\hline \multicolumn{4}{|l|}{ Sex-specific linear regression model } \\
\hline \multicolumn{4}{|l|}{ Model 4} \\
\hline Locomotive activity only (male) & $\mathrm{MET}=0.8766+0.0088 \times \mathrm{ACC}_{\mathrm{fil}}$ & $0.968^{*}$ & 0.634 \\
\hline Locomotive activity only (female) & $\mathrm{MET}=1.3488+0.0083 \times \mathrm{ACC}_{\mathrm{fil}}$ & $0.955^{\star}$ & 0.658 \\
\hline Household activity only (male) & $\mathrm{MET}=1.4022+0.0181 \times \mathrm{ACC}_{\mathrm{fil}}$ & $0.911^{*}$ & 0.446 \\
\hline Household activity only (female) & $\mathrm{MET}=1.3951+0.0195 \times \mathrm{ACC}_{\text {fil }}$ & $0.907^{*}$ & 0.470 \\
\hline \multicolumn{4}{|l|}{ Sex-specific non-linear regression model } \\
\hline \multicolumn{4}{|l|}{ Model 5} \\
\hline Locomotive activity only (male) & $\mathrm{MET}=0.6714+0.0120 \times \mathrm{ACC}_{\mathrm{fil}}^{0.959}$ & $0.968^{*}$ & 0.633 \\
\hline Locomotive activity only (female) & $\mathrm{MET}=0.5367+0.0284 \times \mathrm{ACC}_{\mathrm{fil}}^{0.834}$ & $0.956^{*}$ & 0.654 \\
\hline Household activity only (male) & $\mathrm{MET}=1.3172+0.0254 \times \mathrm{ACC}_{\mathrm{fil}}^{0.939}$ & $0.911^{*}$ & 0.445 \\
\hline Household activity only (female) & $\mathrm{MET}=0.2828+0.2393 \times \mathrm{ACC}_{\mathrm{fil}}^{0.563}$ & $0.915^{\star}$ & 0.451 \\
\hline
\end{tabular}

${ }^{*} P<0.001$.

with combined data of household and locomotive activities had a lower $r$ value compared with all other regressions for locomotive activities only. Regressions for only household activities had slightly lower $r$ values than those for all activities, but the regression standard errors of estimate were improved. Table 4 shows the cross-validation for all regressions. Significant differences were observed between measured values and values estimated from model 1 for most activities. However, models 2-5 accurately estimated the intensity of most household and locomotive activities, with the exceptions of ascending and descending stairs from models 2-5 and normal walking from models 2, 4 and 5, although the differences for normal walking were relatively small. In the cross-validation group, household and locomotive activities were correctly classified $100 \%$ of the time by the $\mathrm{ACC}_{\text {unfil }}: \mathrm{ACC}_{\mathrm{fil}}$ threshold reported previously ${ }^{(27)}$. BlandAltman plots showed that there was improved accuracy of individual activities with models 2-5 compared with model 1 (Fig. 4). Although all models tended to underestimate higher vigorous intensity activity with significant $r^{2}$ values $(P<0.05)$, household activities were clearly well estimated by models $2-5$. The results of the present study remained consistent, whether estimated from linear or non-linear regressions or from sex-specific regressions.

Fig. 5 depicts the relationship between measured MET and $\mathrm{ACC}_{\text {fil }}$ during sedentary activities performed by the validation group. We selected three activities to represent sedentary activities. As shown in Fig. 5, we calculated the regression equation for estimating the intensity of sedentary activities by including dishwashing with the lowest MET on average in our selected household and locomotive activities. The threshold for the classification between sedentary activities and other activities was determined by the point of intersection in the linear regressions for sedentary activities and household activities $(29.9 \mathrm{mG})$. With these threshold and regression equations, resting in the supine position (mean difference 0.04 (SD 0.06) MET, $P<0.01$ ), personal computer work (mean difference - 0.03 (SD 0.09) MET, NS) and dishwashing (mean difference 0.02 (SD 0.31) MET, NS) were estimated adequately in the cross-validation group.

\section{Final model for estimating intensity of physical} activity (n 66)

If $29.9 \mathrm{mG}>\mathrm{ACC}_{\mathrm{fil}}$,

Sedentary activity: MET $=0.8823+0.0351 \times$ ACC $_{\text {fil }}$.

If $29 \cdot 9 \mathrm{mG} \leq \mathrm{ACC}_{\mathrm{fil}}$,

Then if $1 \cdot 16 \leq \mathrm{ACC}_{\text {unfil }}: \mathrm{ACC}_{\text {fil }}$ ratio.

Household activity: $\mathrm{MET}=1 \cdot 3435+0 \cdot 0196 \times \mathrm{ACC}_{\mathrm{fil}}$.

Else if $1 \cdot 16>\mathrm{ACC}_{\text {unfil }}: \mathrm{ACC}_{\text {fil }}$ ratio.

Locomotive activity: $\mathrm{MET}=1 \cdot 1128+0.0086 \times \mathrm{ACC}_{\text {fil }}$.

\section{Discussion}

We have developed a new model to estimate the intensity of daily PA, using a triaxial accelerometer in combination with a novel PA classification algorithm. We classified PA into 
Table 4. Absolute and percentage of differences between measured and estimated metabolic equivalents (MET) from five equation models for household and locomotive activities in the cross-validation group

(Mean values and standard deviations, $n$ 22)

\begin{tabular}{|c|c|c|c|c|c|c|c|c|c|c|c|c|c|c|c|c|c|c|c|c|}
\hline & \multicolumn{4}{|c|}{ Model $1 \dagger$} & \multicolumn{4}{|c|}{ Model 2‡ } & \multicolumn{4}{|c|}{ Model $3 \S$} & \multicolumn{4}{|c|}{ Model 4\| } & \multicolumn{4}{|c|}{ Model 5ף } \\
\hline & \multicolumn{2}{|c|}{$\begin{array}{l}\text { Absolute } \\
\text { difference }\end{array}$} & \multicolumn{2}{|c|}{ \% Difference } & \multicolumn{2}{|c|}{$\begin{array}{l}\text { Absolute } \\
\text { difference }\end{array}$} & \multicolumn{2}{|c|}{ \% Difference } & \multicolumn{2}{|c|}{$\begin{array}{l}\text { Absolute } \\
\text { difference }\end{array}$} & \multicolumn{2}{|c|}{ \% Difference } & \multicolumn{2}{|c|}{$\begin{array}{l}\text { Absolute } \\
\text { difference }\end{array}$} & \multicolumn{2}{|c|}{$\%$ Difference } & \multicolumn{2}{|c|}{$\begin{array}{l}\text { Absolute } \\
\text { difference }\end{array}$} & \multicolumn{2}{|c|}{$\%$ Difference } \\
\hline & Mean & SD & Mean & SD & Mean & SD & Mean & SD & Mean & SD & Mean & SD & Mean & SD & Mean & SD & Mean & SD & Mean & SD \\
\hline Laundry (n 22) & 0.12 & 0.33 & $8 \cdot 3$ & $16 \cdot 0$ & 0.07 & 0.30 & $5 \cdot 3$ & 14.4 & 0.09 & 0.30 & $6 \cdot 0$ & 14.4 & 0.07 & 0.30 & $5 \cdot 4$ & 14.6 & 0.09 & 0.31 & $6 \cdot 1$ & $15 \cdot 0$ \\
\hline $\begin{array}{l}\text { Dishwashing ( } n \\
\text { 21) }\end{array}$ & 0.36 & 0.27 & $23 \cdot 7^{\star \star \star}$ & 21.3 & 0.11 & 0.27 & $9 \cdot 0$ & $19 \cdot 1$ & 0.03 & 0.29 & 3.8 & 19.6 & 0.11 & 0.27 & 8.8 & $19 \cdot 0$ & 0.03 & 0.31 & 3.9 & 20.6 \\
\hline $\begin{array}{l}\text { Moving a small } \\
\text { load ( } n \text { 22) }\end{array}$ & -1.46 & 0.72 & $-30 \cdot 4^{\star \star \star}$ & $10 \cdot 3$ & -0.22 & 0.69 & -3.0 & 14.4 & -0.25 & 0.70 & -3.5 & $14 \cdot 3$ & -0.22 & 0.72 & $-2 \cdot 7$ & 14.8 & -0.23 & 0.72 & $-2 \cdot 9$ & $14 \cdot 7$ \\
\hline Vacuuming (n 22) & -0.46 & 0.73 & $-10 \cdot 4^{\star \star}$ & 19.7 & -0.05 & 0.64 & $3 \cdot 0$ & $22 \cdot 2$ & 0.04 & 0.64 & $6 \cdot 2$ & $23 \cdot 0$ & -0.05 & 0.64 & $3 \cdot 1$ & 21.9 & 0.04 & 0.65 & $6 \cdot 0$ & $22 \cdot 4$ \\
\hline $\begin{array}{l}\text { Slow walking ( } \\
\text { 21) }\end{array}$ & 0.63 & 0.42 & $21 \cdot 5^{\star \star \star}$ & $14 \cdot 8$ & 0.10 & 0.45 & 4.2 & 14.6 & 0.07 & 0.47 & $3 \cdot 2$ & $15 \cdot 0$ & 0.12 & 0.41 & 4.8 & 13.6 & 0.06 & 0.44 & $2 \cdot 9$ & $14 \cdot 3$ \\
\hline $\begin{array}{l}\text { Normal } \\
\quad \text { walking ( }(n 21)\end{array}$ & 0.67 & 0.48 & $19 \cdot 8^{\star \star \star}$ & $15 \cdot 1$ & 0.23 & 0.50 & $7 \cdot 6^{\star}$ & $14 \cdot 3$ & 0.22 & 0.50 & 7.4 & $14 \cdot 4$ & 0.22 & 0.48 & $7 \cdot 4^{\star}$ & 13.7 & 0.23 & 0.48 & $7 \cdot 7^{\star}$ & 14.0 \\
\hline $\begin{array}{l}\text { Brisk walking } \\
\quad(n \text { 22) }\end{array}$ & 0.34 & 0.70 & $9 \cdot 1$ & $15 \cdot 7$ & 0.03 & 0.72 & $2 \cdot 4$ & $15 \cdot 1$ & 0.04 & 0.72 & $2 \cdot 6$ & $15 \cdot 2$ & 0.04 & 0.69 & 2.5 & 14.7 & 0.09 & 0.69 & $3 \cdot 6$ & $15 \cdot 0$ \\
\hline $\begin{array}{l}\text { Walking while } \\
\text { carrying a bag } \\
(n 22)\end{array}$ & 0.34 & 0.59 & $9 \cdot 8^{*}$ & $15 \cdot 3$ & -0.06 & 0.61 & 0.1 & 14.6 & -0.06 & 0.61 & 0.1 & 14.7 & -0.06 & 0.57 & 0.1 & 13.8 & -0.03 & 0.58 & 0.7 & 14.1 \\
\hline Jogging (n 20) & -0.50 & 1.39 & -3.8 & 13.9 & -0.18 & 1.44 & -0.4 & 14.9 & -0.23 & 1.43 & -0.9 & 14.7 & -0.17 & 1.42 & -0.3 & 14.7 & -0.19 & 1.38 & -0.6 & $14 \cdot 3$ \\
\hline $\begin{array}{l}\text { Ascending stairs } \\
\quad(n 19)\end{array}$ & $-4 \cdot 13$ & 0.78 & $-53 \cdot 3^{\star \star \star}$ & 4.9 & -4.69 & 0.78 & $-60 \cdot 6^{\star \star \star}$ & 4.5 & -4.73 & 0.78 & $-61 \cdot 2^{\star \star \star}$ & 4.6 & $-4 \cdot 68$ & 0.81 & $-60 \cdot 5^{\star \star \star}$ & 4.8 & -4.75 & 0.80 & $-61 \cdot 4^{\star \star \star}$ & 4.7 \\
\hline $\begin{array}{l}\text { Descending stairs } \\
\quad(n \text { 20) }\end{array}$ & $1 \cdot 13$ & 0.73 & $40 \cdot 7^{\star \star \star}$ & $30 \cdot 0$ & 0.68 & 0.78 & $25 \cdot 6^{\star \star}$ & 29.2 & 0.66 & 0.79 & $25 \cdot 1^{\star \star}$ & 29.5 & 0.70 & 0.79 & $26 \cdot 2^{\star \star}$ & 28.9 & 0.69 & 0.81 & $26 \cdot 1^{\star \star}$ & 29.6 \\
\hline
\end{tabular}

Mean values were significantly different compared with measured MET: ${ }^{\star} P<0.05,{ }^{* \star} P<0.01,{ }^{\star \star *} P<0.001$.

† Linear regression model for estimating locomotive and household activities together.

¥ Linear regression model for estimating locomotive and household activities separately.

$\S$ Non-linear regression model for estimating locomotive and household activities separately.

Sex-specific linear regression model for estimating locomotive and household activities separately.

I Sex-specific non-linear regression model for estimating locomotive and household activities separately. 

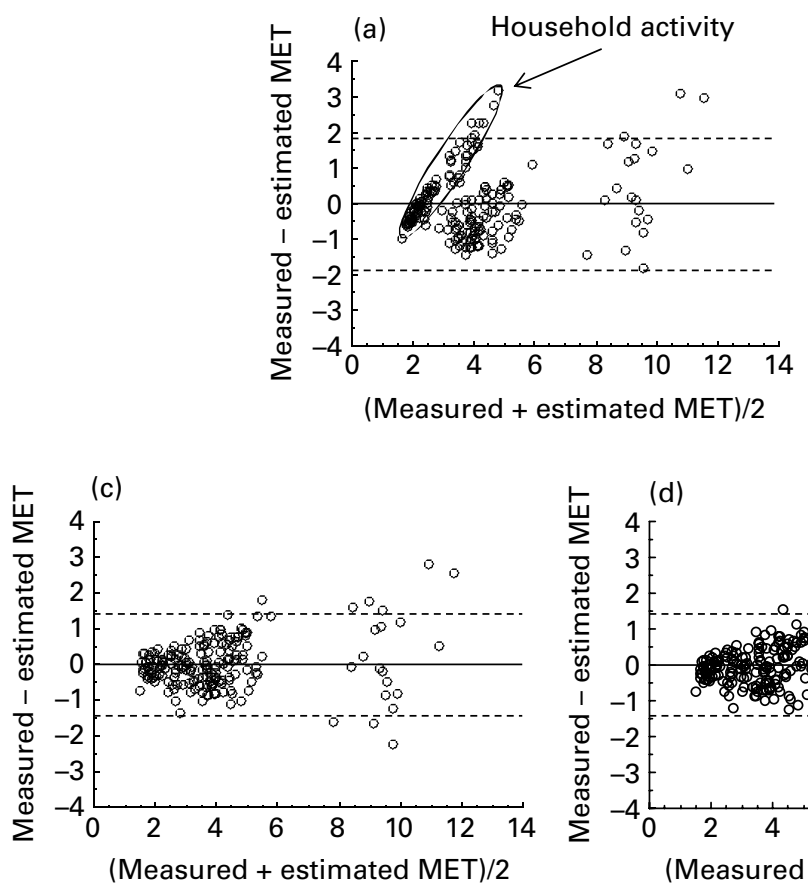

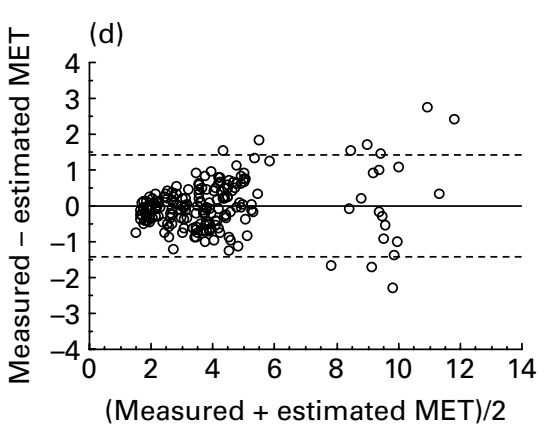

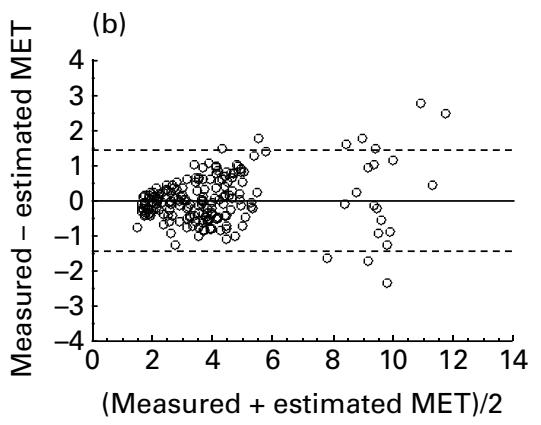

(e)

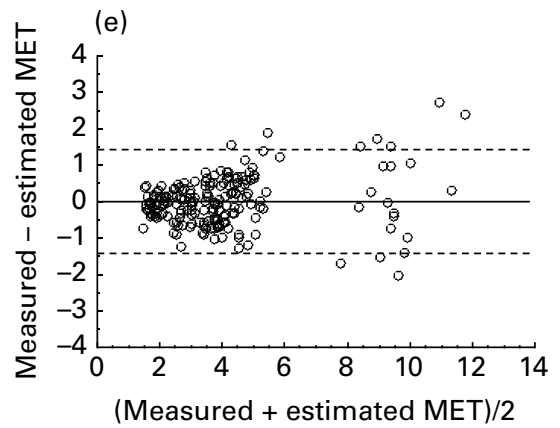

Fig. 4. Bland-Altman analysis. Differences between measured and estimated metabolic equivalents (MET) are plotted against measured and estimated mean MET for household and locomotive activities. (a) Model 1, linear regression model for estimating locomotive and household activities together ( $r$ 0.237); (b) model 2 , linear regression model for estimating locomotive and household activities separately ( $r$ 0.207); (c) model 3, non-linear regression model for estimating locomotive and household activities separately ( $r 0.219)$; (d) model 4, sex-specific linear regression model for estimating locomotive and household activities separately ( $r$ 0.212); (e) model 5, sex-specific non-linear regression model for estimating locomotive and household activities separately ( $r 0.207)$. -, Mean; ---, 95\% Cl of the observations.

locomotive, household and sedentary activities with thresholds determined by the $\mathrm{ACC}_{\text {unfil }}: \mathrm{ACC}_{\mathrm{fil}}$ ratio (GRPACA) or accelerometer counts ${ }^{(27)}$. The rate of correct classification was excellent: $100 \%$ of the activities performed by our subjects were correctly classified as locomotive or household. With our new classification algorithm, the regressions clearly improved the accuracy of estimating the intensity of various PA, compared with a non-classification model. This novel method is capable of estimating the intensity of PA accurately and immediately, serving as a practical field tool for researchers as well as for general users.

In agreement with previous studies ${ }^{(18-21,31)}$, we observed that the multiple equation model improved the accuracy of estimating household and locomotive activity intensities, compared with the one-equation model; accuracy improvements occurred for household activities in particular. With the exceptions of ascending and descending stairs, average percentage differences were within $10 \%$ in the two-equation model, with more than $10 \%$ differences in several activities in the one-equation model. Furthermore, we attempted to estimate the intensity of PA with non-linear regression and sex-specific regression (or non-regression) models. Prediction errors obtained from the linear and non-linear regression models were comparable in the present study (Table 4). While it is still controversial whether the linear or non-linear regression model is a better predictive model $^{(8)}$, inclusion of the GRPACA did not necessitate non-linear or sex-specific regression equations. To our knowledge, there is no evidence of a quadratic relationship between MET and accelerometer counts in various PA. Therefore, the linear regression model may obtain comparable predictions as the non-linear regression model in the present study, under actual freeliving conditions. Furthermore, the sex-specific equation model did not provide a more accurate estimation (Table 4), indicating that we have developed new equations by linear regressions without taking sex into account.

Accurate estimation of sedentary activities is important, as many people perform sedentary activities at least several hours/ $\mathrm{d}^{(32,33)}$. Previously developed accelerometer-based models overestimate the intensity of sedentary activities ${ }^{(16)}$. In the present study, sedentary activities clearly had lower accelerometer counts than other activities. Initially, we hypothesised that the cut-off threshold between intensities of sedentary and other activities should be the midpoint of the highest sedentary accelerometer count and the lowest accelerometer count from the other activities. However, in the present study, we observed a small gap between sedentary and household activities in the relationship between MET and ACC $_{\text {fil }}$. Therefore, we developed the equation for sedentary activities by including dishwashing, which displayed the lowest accelerometer counts of our household or locomotive activity. Using this consideration, activities about 1.5 MET could be estimated accurately. Therefore, we have classified an activity of less than 2 MET as a sedentary activity, using a cut-off threshold determined by accelerometer counts.

Although PA intensity estimates were improved with our model, we could not directly compare the present results with previously reported models designed for data collecting 


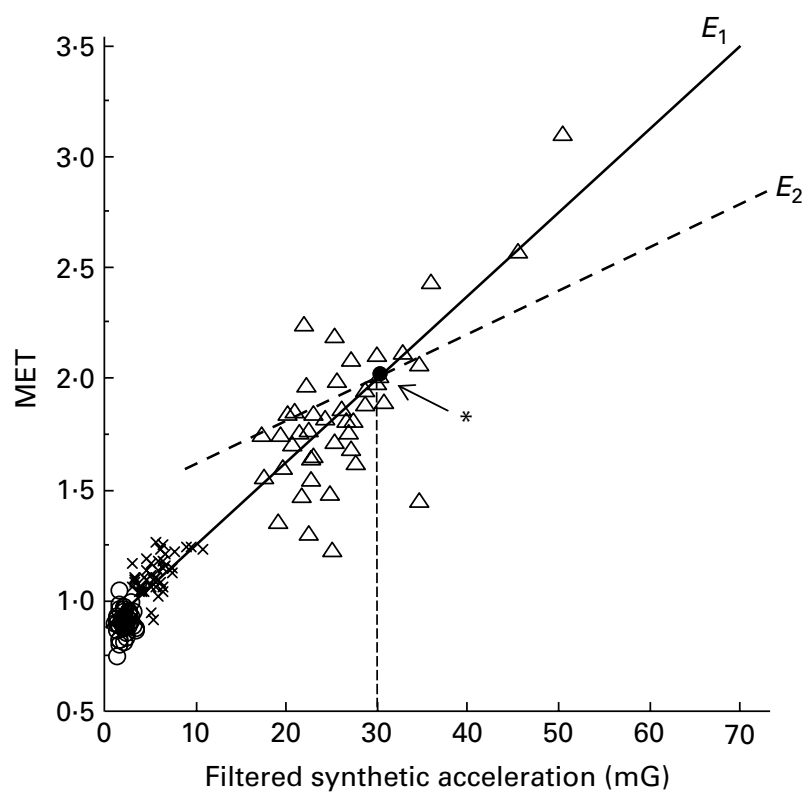

Fig. 5. Relationship between measured metabolic equivalents (MET) and filtered synthetic accelerations during sedentary activities in the validation group ( $n$ 44). $E_{1}$ ( $r 0.942, P<0.001$, standard error of estimate $0.151 \mathrm{MET}$ ), regression line for sedentary activities; $E_{2}$, regression line for household activities. * Threshold point for the classification between sedentary and household activities $(29.9 \mathrm{mG})$. Dishwashing was included in both $E_{1}$ and $E_{2}$. $O$, Resting in the supine position; $X$, personal computer work; $\Delta$, dishwashing.

and developing equations. However, our accuracies for some activities, such as personal computer work, vacuuming and dishwashing, are slightly better than the results obtained by Crouter et al. ${ }^{(18-21,34)}$, who used two equations with a classification algorithm based on the $\mathrm{CV}$ of the acceleration count. Moreover, our model possesses the following advantages over previous models: (1) our classification algorithm is accurate but simple, leading to immediate estimation of PA intensity following a long period of data collection; (2) our measuring device is secured to the waist by a clip only; (3) the Douglas bag method, not a portable analyser, was used as the reference method; (4) MET were calculated with measured RMR (not $3.5 \mathrm{ml} / \mathrm{kg}$ per $\min$ or $4.2 \mathrm{~kJ} / \mathrm{kg}$ per $\mathrm{h}$ (1 kcal/kg per h)); (5) values from a triaxial accelerometer, not a uniaxial accelerometer, were used for developing equations.

Several algorithms have been developed for PA classification. These algorithms were constructed using the CV of the acceleration count based on the ActiGraph or Actical devices $^{(18-21,34)}$ or using the ratio of vertical acceleration counts to horizontal acceleration counts based on the ActivTracer device ${ }^{(22,25)}$. In these studies, the percentage of correct classifications does not seem to be high, even for the subjects used in the classification development. Our algorithm may classify locomotive and household activities with higher accuracy. On the other hand, other reported classification algorithms $^{(26,35,36)}$ were developed to divide PA into further subtypes. These additional divisions require a large quantity of data, a complex calculation process or the placement of sensors over the whole body; it is difficult to maintain battery power over long periods, to check PA intensities in real time and to wear and remove the device easily. Our device is worn just on the waist, is held by a clip and PA intensities were displayed immediately. This unique device is useful for applied researchers or professional health advisers to investigate PA in the field, and general users can monitor their activity status by themselves, as the commercial product has an liquid crystal display that can indicate real-time MET values or step counts.

We employed the Douglas bag method as a reference for measuring EE, while previous studies used a portable metabolic system such as Aerosport TEEM 100 or COSMED K4b2. For these portable metabolic systems, validation of assessing EE during PA has been reported ${ }^{(37-40)}$. A portable metabolic system also has the advantage of measuring various dynamic activities outdoors. However, portable metabolic systems slightly overestimate or underestimate $\mathrm{O}_{2}$ uptake during exercise testing, compared with reference methods ${ }^{(40-43)}$. Therefore, the Douglas bag method may be preferable to a portable metabolic system as a reference method for measuring EE during various types of PA

Whether measured values or a constant value of $3.5 \mathrm{ml} / \mathrm{kg}$ should be used for the RMR value of 1 MET is debatable. Typical values for the normal-weight population were $3.5 \mathrm{ml} / \mathrm{kg}$ per min and $4.2 \mathrm{~kJ} / \mathrm{kg}$ per h $(1 \mathrm{kcal} / \mathrm{kg}$ per h). However, average measured RMR were much lower than $3.5 \mathrm{ml} / \mathrm{kg}$ per min or $4.2 \mathrm{~kJ} / \mathrm{kg}$ per h $(1 \mathrm{kcal} / \mathrm{kg}$ per h) in 671 subjects, although many were overweight or obese ${ }^{(44)}$. In particular, body composition contributed to the variance in RMR. In the present study, the average RMR value was $4.1 \mathrm{~kJ} / \mathrm{kg}$ per $\mathrm{h}$ $(0.99 \mathrm{kcal} / \mathrm{kg}$ per $\mathrm{h})$, but the standard deviation was relatively large $(0.8 \mathrm{~kJ} / \mathrm{kg}$ per h $(0.19 \mathrm{kcal} / \mathrm{kg}$ per h)). To our knowledge, no description exists of whether the RMR value of $3.5 \mathrm{ml} / \mathrm{kg}$ per min was previously measured in a fasting state ${ }^{(45)}$, although the present study and Byrne et al. ${ }^{(44)}$ measured RMR in the fasting state. Therefore, the use of measured RMR as 1 MET could lead to increased accuracy of estimating the intensity of PA.

A triaxial accelerometer, capable of measuring both vertical and horizontal accelerations, is more informative than a uniaxial accelerometer, possibly permitting more accurate estimates of PA intensities. However, previous studies ${ }^{(16,25,46)}$ have reported that the accuracy of estimating PA intensities did not differ between triaxial and uniaxial accelerometers if these values were estimated by a one-equation model. Although our classification algorithm can calculate the cutoff threshold even using a uniaxial accelerometer count, we confirmed that the classification developed with a synthetic accelerometer count is more accurate than that based only on a vertical (uniaxial) accelerometer count. Therefore, our estimation by triaxial accelerometer counts should lead to increased accuracy compared with a uniaxial accelerometer.

The present study had several limitations. We could not accurately estimate the intensity of ascending and descending stairs. Although previous studies ${ }^{(18-21,36)}$ have estimated the intensities of these activities relatively well, validity was assessed by a condition combining ascension and descension of stairs, with only Yamazaki et al. ${ }^{(47)}$ performing the individual 
assessments. Under daily living conditions, ascending and descending stairs are normally performed separately, and thus these activities should be assessed separately. In addition, we did not include stationary ergometer or cycling in the present study. Furthermore, the developed model tended to underestimate higher vigorous intensity activity. Therefore, future studies are needed using the doubly labelled water method or a metabolic chamber to investigate the validity of our model. In addition, studies are needed to compare our accelerometer with other types of accelerometers under freeliving conditions. Furthermore, more investigation is needed to determine how well the model developed in the present study applies to other populations such as obese individuals or children.

We have recently reported a simple but accurate classification algorithm to differentiate between locomotive and household activities, with a cut-off determined by the $\mathrm{ACC}_{\text {unfil }}$ : $\mathrm{ACC}_{\text {fil }}$ ratio ${ }^{(27)}$. Additionally, sedentary activities could be discriminated from household and locomotive activities with accelerometer counts. With this classification algorithm, our new model exhibited improved accuracy in estimating the intensity of various PA, compared with non-classification models. Furthermore, this new model is capable of estimating PA intensity immediately. Therefore, the method is useful for field investigations by scientists as well as for self-monitoring of activity by the general public.

\section{Acknowledgements}

The present study was supported by the Health and Labor Sciences Research Grants for Comprehensive Research on Cardiovascular and Life-Style Related Diseases from the Japanese Ministry of Health, Labor and Welfare (principal investigator: S. T.). Heartfelt thanks are due to the subjects who participated in the present study. We wish to thank the members of the National Institute of Health and Nutrition and Omron Healthcare Company Limited, especially Hiroko Kogure, Emiko Taguri, Rieko Miyake, Ryosuke Doi and Kaori Kawaguchi for their help in data acquisition and analysis. K. O., S. T., Y. O., Y. H., K. I.-T. and I. T. designed the study; K. O., S. T., Y. O., Y. H. and K. I.-T. performed the experiments; K. O., S. T. and Y. O. analysed the data; K. O., S. T. and Y. O. wrote a draft of the manuscript; Y. H., K. I.-T. and I. T. reviewed and edited the manuscript. None of the authors had a conflict of interest.

\section{References}

1. US Department of Health \& Human Services (2008) Physical Activity Guidelines Advisory Committee Report 2008. http:// www.health.gov/PAGuidelines/Report/.

2. Saris WH, Blair SN, van Baak MA, et al. (2003) How much physical activity is enough to prevent unhealthy weight gain? Outcome of the IASO 1st Stock Conference and consensus statement. Obes Rev 4, 101-114.

3. Levine JA, Eberhardt NL \& Jensen MD (1999) Role of nonexercise activity thermogenesis in resistance to fat gain in humans. Science 283, 212-214.
4. Levine JA, Lanningham-Foster LM, McCrady SK, et al. (2005) Interindividual variation in posture allocation: possible role in human obesity. Science 307, 584-586.

5. Dale D, Welk GJ \& Matthews CE (2002) Methods for assessing physical activity and challenges for research. In Physical Activity Assessments for Health-related Research, pp. 19-34 [GJ Welk, editor]. Champaign, IL: Human Kinetics Publishers.

6. Melanson EL Jr \& Freedson PS (1996) Physical activity assessment: a review of methods. Crit Rev Food Sci Nutr 36, 385-396.

7. Neilson HK, Robson PJ, Friedenreich CM, et al. (2008) Estimating activity energy expenditure: how valid are physical activity questionnaires? Am J Clin Nutr 87, 279-291.

8. Chen KY \& Bassett DR Jr (2005) The technology of accelerometry-based activity monitors: current and future. Med Sci Sports Exerc 37, S490-S500.

9. Trost SG, McIver KL \& Pate RR (2005) Conducting accelerometer-based activity assessments in field-based research. Med Sci Sports Exerc 37, S531-S543.

10. Ward DS, Evenson KR, Vaughn A, et al. (2005) Accelerometer use in physical activity: best practices and research recommendations. Med Sci Sports Exerc 37, S582-S588.

11. Richardson CR, Newton TL, Abraham JJ, et al. (2008) A metaanalysis of pedometer-based walking interventions and weight loss. Ann Fam Med 6, 69-77.

12. Bravata DM, Smith-Spangler C, Sundaram V, et al. (2007) Using pedometers to increase physical activity and improve health: a systematic review. JAMA 298, 2296-2304.

13. Bassett DR Jr, Ainsworth BE, Swartz AM, et al. (2000) Validity of four motion sensors in measuring moderate intensity physical activity. Med Sci Sports Exerc 32, S471-S480.

14. Matthews CE (2005) Calibration of accelerometer output for adults. Med Sci Sports Exerc 37, S512-S522.

15. Welk GJ, Blair SN, Wood K, et al. (2000) A comparative evaluation of three accelerometry-based physical activity monitors. Med Sci Sports Exerc 32, S489-S497.

16. Rothney MP, Schaefer EV, Neumann MM, et al. (2008) Validity of physical activity intensity predictions by ActiGraph, Actical, and RT3 accelerometers. Obesity (Silver Spring) 16, $1946-1952$

17. Chen KY \& Sun M (1997) Improving energy expenditure estimation by using a triaxial accelerometer. J Appl Physiol 83, 2112-2122.

18. Crouter SE \& Bassett DR Jr (2008) A new 2-regression model for the Actical accelerometer. Br J Sports Med 42, 217-224.

19. Crouter SE, Churilla JR, Bassett DR, et al. (2006) Estimating energy expenditure using accelerometers. Eur J Appl Physiol 98, 601-612.

20. Crouter SE, Churilla JR \& Bassett DR Jr (2008) Accuracy of the Actiheart for the assessment of energy expenditure in adults. Eur J Clin Nutr 62, 704-711.

21. Crouter SE, Clowers KG \& Bassett DR Jr (2006) A novel method for using accelerometer data to predict energy expenditure. J Appl Physiol 100, 1324-1331.

22. Midorikawa T, Tanaka S, Kaneko K, et al. (2007) Evaluation of low-intensity physical activity by triaxial accelerometry. Obesity (Silver Spring) 15, 3031-3038.

23. Pober DM, Staudenmayer J, Raphael C, et al. (2006) Development of novel techniques to classify physical activity mode using accelerometers. Med Sci Sports Exerc $\mathbf{3 8}$, 1626-1634.

24. Rothney MP, Neumann M, Beziat A, et al. (2007) An artificial neural network model of energy expenditure using nonintegrated acceleration signals. J Appl Physiol 103, 1419-1427. 
25. Tanaka C, Tanaka S, Kawahara J, et al. (2007) Triaxial accelerometry for assessment of physical activity in young children. Obesity (Silver Spring) 15, 1233-1241.

26. Zhang K, Werner P, Sun M, et al. (2003) Measurement of human daily physical activity. Obes Res 11, 33-40.

27. Oshima Y, Kawaguchi K, Tanaka S, et al. (2010) Classifying household and locomotive activities using a triaxial accelerometer. Gait Posture 31, 370-374.

28. Ohkawara K, Tanaka S, Ishikawa-Takata K, et al. (2008) Twenty-four-hour analysis of elevated energy expenditure after physical activity in a metabolic chamber: models of daily total energy expenditure. Am J Clin Nutr 87, $1268-1276$

29. Weir JB (1949) New methods for calculating metabolic rate with special reference to protein metabolism. J Physiol 109, $1-9$.

30. Bland JM \& Altman DG (1986) Statistical methods for assessing agreement between two methods of clinical measurement. Lancet 1, 307-310.

31. Corder K, Brage S, Mattocks C, et al. (2007) Comparison of two methods to assess PAEE during six activities in children. Med Sci Sports Exerc 39, 2180-2188.

32. Institute of Medicine (2005) Dietary Reference Intakes for Energy, Carbohydrate, Fiber, Fat, Fatty, Acids, Cholesterol, Protein and Amino Acids. Washington, DC: The National Academies Press.

33. Westerterp KR (2001) Pattern and intensity of physical activity. Nature 410, 539

34. Crouter SE, Kuffel E, Haas JD, et al. (2010) Refined tworegression model for the ActiGraph accelerometer. Med Sci Sports Exerc 42, 1029-1037.

35. Bonomi AG, Plasqui G, Goris AH, et al. (2009) Improving assessment of daily energy expenditure by identifying types of physical activity with a single accelerometer. $J$ Appl Physiol 107, 655-661.

36. Staudenmayer J, Pober D, Crouter S, et al. (2009) An artificial neural network to estimate physical activity energy expendi- ture and identify physical activity type from an accelerometer. J Appl Physiol 107, 1300-1307.

37. Maiolo C, Melchiorri G, Iacopino L, et al. (2003) Physical activity energy expenditure measured using a portable telemetric device in comparison with a mass spectrometer. $\mathrm{Br}$ J Sports Med 37, 445-447.

38. McLaughlin JE, King GA, Howley ET, et al. (2001) Validation of the COSMED K4 b2 portable metabolic system. Int J Sports Med 22, 280-284.

39. Melanson EL, Freedson PS, Hendelman D, et al. (1996) Reliability and validity of a portable metabolic measurement system. Can J Appl Physiol 21, 109-119.

40. Wideman L, Stoudemire NM, Pass KA, et al. (1996) Assessment of the aerosport TEEM 100 portable metabolic measurement system. Med Sci Sports Exerc 28, 509-515.

41. Duffield R, Dawson B, Pinnington HC, et al. (2004) Accuracy and reliability of a Cosmed $\mathrm{K} 4 \mathrm{~b} 2$ portable gas analysis system. J Sci Med Sport 7, 11-22.

42. Littlewood RA, White MS, Bell KL, et al. (2002) Comparison of the Cosmed K4 b(2) and the Deltatrac II metabolic cart in measuring resting energy expenditure in adults. Clin Nutr 21, 491-497.

43. Mc Naughton LR, Sherman R, Roberts S, et al. (2005) Portable gas analyser Cosmed K4b2 compared to a laboratory based mass spectrometer system. J Sports Med Phys Fitness 45, 315-323.

44. Byrne NM, Hills AP, Hunter GR, et al. (2005) Metabolic equivalent: one size does not fit all. J Appl Physiol 99, 1112-1119.

45. Jette M, Sidney K \& Blumchen G (1990) Metabolic equivalents (METS) in exercise testing, exercise prescription, and evaluation of functional capacity. Clin Cardiol 13, 555-565.

46. Leenders NY, Sherman WM \& Nagaraja HN (2006) Energy expenditure estimated by accelerometry and doubly labeled water: do they agree? Med Sci Sports Exerc 38, 2165-2172.

47. Yamazaki T, Gen-No H, Kamijo Y, et al. (2009) A new device to estimate $\mathrm{VO}_{2}$ during incline walking by accelerometry and barometry. Med Sci Sports Exerc 41, 2213-2219. 